\title{
Sensitivity-Specificity Tradeoff for Different Alarm Strategies in Continuous 12-Lead ST-Segment Monitoring
}

\author{
JY Wang ${ }^{1}, \mathrm{JW}$ Warren ${ }^{2}$, BM Horáček ${ }^{2}$ \\ ${ }^{1}$ Philips Medical Systems, Andover, MA, USA \\ ${ }^{2}$ Dalhousie University, Halifax, NS, Canada
}

\begin{abstract}
Our objective was to assess both the sensitivity and specificity of various alarm-detection strategies for realtime ST-segment monitoring. The dataset was comprised of ST measurements from 12-lead Mason-Likar electrocardiograms (ML ECG) obtained from 88 patients before and during elective balloon-inflation angioplasty. We compared the ability of the 12-lead ML ECG and its subsets to detect ischemia, based on an index derived from the absolute values of the ST shift in "non-ischemic" and "ischemic" states. The sensitivity and specificity of a classifier using this index for a range of threshold values yielded receiver operating characteristic (ROC) curves. Detection performance was assessed as mean area under 1,000 ROC curves (AUC) generated by a bootstrap method with replacement. Results show that the reduced lead sets with 1 limb lead and 2 precordial leads (e.g. III, V3, V6 or III, V1, V4) attain AUC values that are not significantly different from those of the 12-lead ML ECG.
\end{abstract}

\section{Introduction}

Electrocardiographic ST-segment monitoring is the only technique that allows continuous non-invasive tracking of evolving ischemic events in patients with acute coronary syndromes. Current bedside monitors are capable of providing up to 12 ECG leads for continuous monitoring. The presence of an ST-segment deviation of $100 \mu \mathrm{V}$ or greater in at least one of the standard $12 \mathrm{ECG}$ leads is the current norm for ischemia detection [1,2]. At this threshold, detection of ischemia is sensitive, but it also has the potential of generating many false alarms when used in a real-time monitoring environment $[3,4]$. Strategies for reducing the number of false alarms-such as using higher detection threshold or requiring that multiple leads exceed the threshold-have been implemented [2,5], but their sensitivity and specificity in detecting ischemic ST-segment changes have not been evaluated. Therefore, we sought to directly compare the abilities of multilead alarm strategies to detect STsegment changes associated with acute ischemia.

\section{Methods}

\subsection{Database}

The 12-lead ST measurements for ML ECG were extracted from the Dalhousie PTCA database, containing body-surface potential maps (BSPMs) of patients with single-vessel coronary artery disease for which they underwent elective balloon-inflation angioplasty [6]. We used data from 88 of these patients: 31 whose lesion was in the left anterior descending coronary artery, 22 whose lesion was in the left circumflex coronary artery, and 35 whose lesion was in the right coronary artery. The original data [6] were recorded simultaneously from 120 ECG leads for each patient; the lead array had 3 limb leads (at the Mason-Likar electrode positions [7]) and 117 unipolar chest leads. Data processing involved averaging ECGs for 15-sec episodes at the "non-ischemic" state (before balloon inflation) and at the "ischemic" state (after $60 \mathrm{sec}$ of inflation). The ST shift (relative to the TP baseline) was then determined from the averaged complexes at $60 \mathrm{~ms}$ after the $\mathrm{J}$ point. Patients whose BSPMs showed a discernible pattern of ST shift during peak balloon inflation were designated as "responders"; there were 45 such patients - 15 for each coronary artery occluded [6]. Fig. 1 shows ST shifts for the entire group and for "responders" only.

\subsection{Assessment of ST alarm strategies}

We assessed the various alarm strategies' sensitivity and specificity in detecting ischemia as a function of the threshold of an ischemic index computed from the absolute values of the ST-segment shift, $|\mathrm{ST}|$, in all constituent leads of the given lead set. Using all 12 leads of the ML ECG, we first calculated $\Sigma \mid$ STI (denoted as ST12). We then considered strategies requiring that any 1 $|\mathrm{ST}|$ value (or any $2|\mathrm{ST}|$ values, any $3|\mathrm{ST}|$ values, $|\mathrm{ST}|$ values in any 2 contiguous leads) of all 12 leads of the ML ECG exceed the threshold. Finally, calculating $\Sigma|S T|$ again as a parameter, we considered subsets of the ML ECG: the 12 individual leads, 66 pairs, and 220 triplets. 
Therefore, a total of 303 alarm strategies were assessed and ranked with regard to their comparative merits in detecting ischemia.
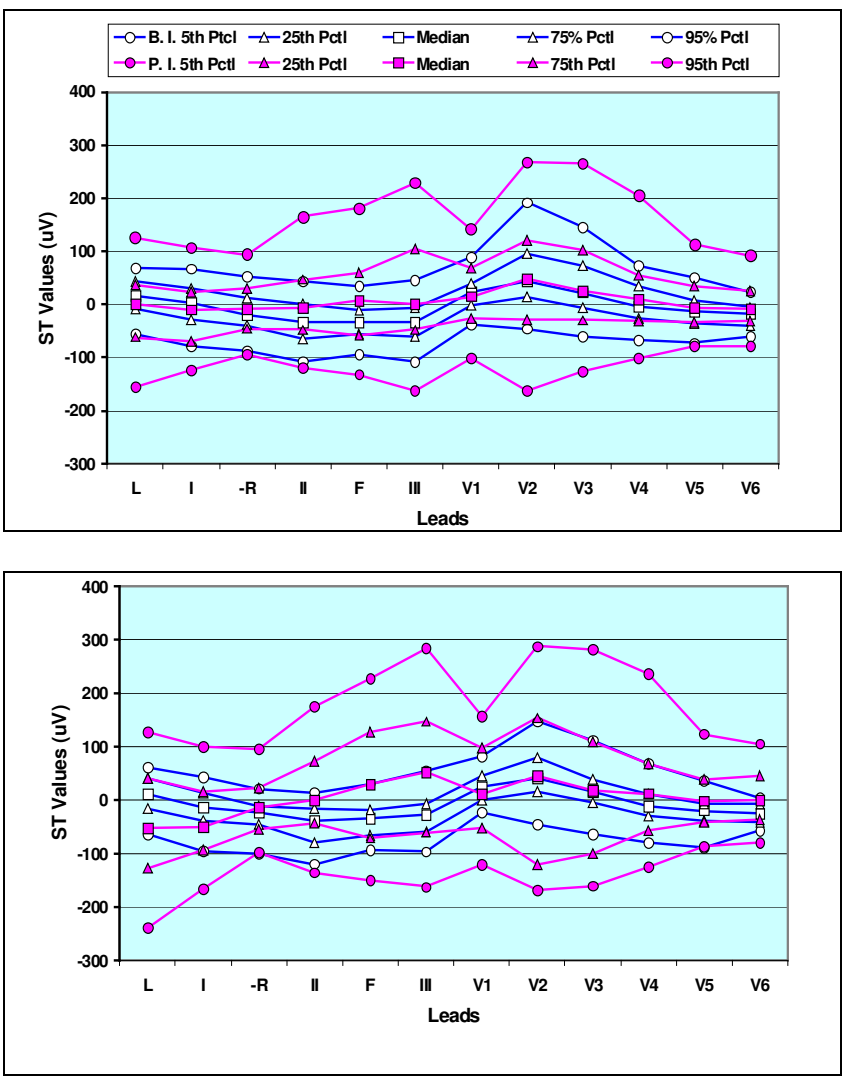

Figure 1. ST shifts in 12-lead ECG for all 88 patients from the Dalhousie database (top) and for the subgroup of 45 "responders" (bottom). Median, $5^{\text {th }}, 25^{\text {th }}, 75^{\text {th }}$, and $95^{\text {th }}$ percentiles are shown before inflation (B.I.) as open symbols, and at peak inflation (P.I.) as solid symbols.

\subsection{Performance Criteria}

The ability of the different lead sets to detect ischemia was assessed as follows: We performed classification that separated the "ischemic" from the "non-ischemic" state using the alarm strategies described in the previous paragraph, with the variable threshold value for the entire range of sensitivity and specificity. This allowed us to obtain the receiver operating characteristic (ROC) curves [8] and an "optimal" threshold value (defined here as a point on the ROC curve for which sensitivity and specificity are equal). Using a bootstrap method with replacement (for $n=88$ and $n=45$ ), we generated ROC curves for 1,000 trials [9]; the mean value of the area under the ROC curve (AUC) and the mean value of an "optimal" threshold were determined. The mean AUC value was then used as a measure of the lead set's ability to distinguish the "ischemic" state from the "nonischemic" state [8]. This entire procedure was performed for each of the 303 lead sets separately, and the lead sets were ranked based on the AUC value.

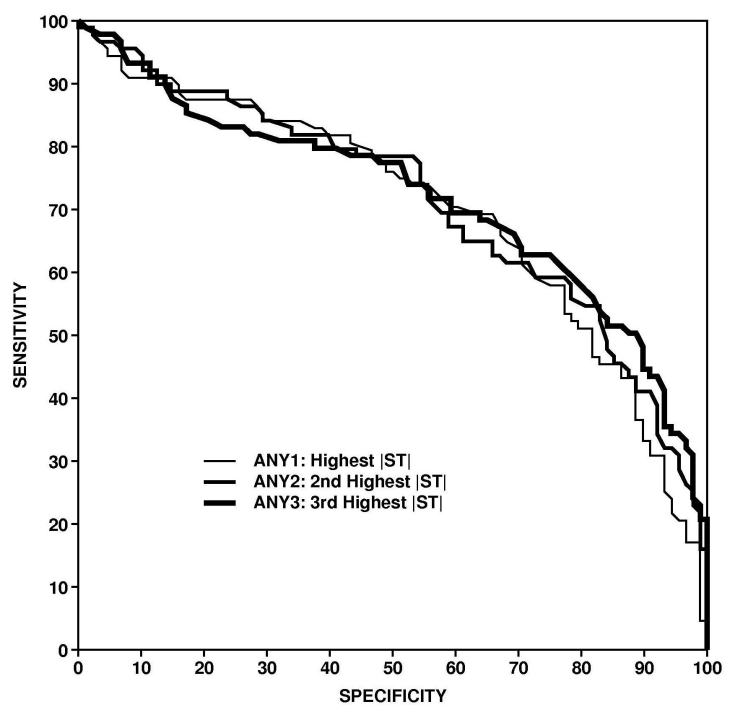

Figure 2. ROC curves indicating detection performance of three alarm strategies for all 88 patients from the Dalhousie database. Thick line, ROC for ischemia detection using any three of the 12 leads of the ML ECG; line of medium thickness, ROC for ischemia detection using any two of 12 leads; thin line, ROC for ischemia detection using any one of 12 leads.

\section{Results}

Using the ST measurements in the 12 leads of the ML ECG for the entire study group and for the "responders" subgroup as input data (Fig. 1), we generated and plotted mean ROC curves, such as those in Fig. 2, for all lead sets. The mean AUCs and the mean "optimal" thresholds with their standard deviations (SD) were calculated for 1,000 bootstrap trials, and lead sets were ranked. A summary of the AUC values for the top-ranked lead sets in the 5 lead groups is presented in Fig. 3; this figure shows that the AUC values for all lead groups except Lead Group 1 form "tight" clusters and that the differences in these values are not significant (NS) in each cluster. For the entire patient population $(n=88)$, notable top-ranked lead sets of all 5 lead groups are listed with AUCs and "optimal" thresholds in Tables 1-3. Results for Lead Group 5 are presented both for the entire patient population (Table 3 ) and for the "responders" only (Table 4) to allow comparison. 


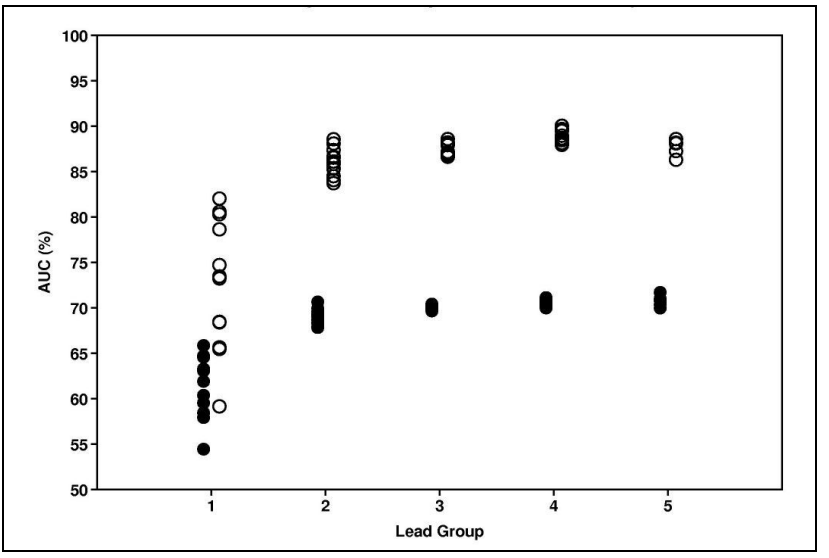

Figure 3. Performance ranking based on mean AUC for subsets of ML ECG divided into 5 lead groups. Group 1, single leads; Group 2, pairs of leads; Group 3, triplets with 2 limb leads and 1 precordial lead; Group 4, triplets with $1 \mathrm{limb}$ lead and 2 precordial leads; Group 5, all 12 leads. Solid circles, entire group $(n=88)$; open circles, "responders" only $(\mathrm{n}=45)$.

Table 1 shows that the single leads of the 12-lead ML ECG rank low among all 303 lead sets. The values of the "optimal" threshold are very low; consequently, alarm strategies relying on any single (fixed) lead would be prone to false alarms. Nevertheless, this table provides valuable clues regarding the leads' relative worth.

Table 1. Performance of individual leads of the ML ECG (Lead Group 1).

\begin{tabular}{lccc}
\hline Lead & AUC \pm SD $(\%)$ & Th \pm SD $(\mu \mathrm{V})^{*}$ & Rank \\
\hline III & $65.55 \pm 3.68$ & $46 \pm 5$ & 213 \\
V3 & $65.13 \pm 3.36$ & $51 \pm 6$ & 241 \\
aVL & $64.72 \pm 4.09$ & $37 \pm 4$ & 254 \\
V4 & $63.61 \pm 3.97$ & $36 \pm 3$ & 274 \\
aVF & $63.51 \pm 3.82$ & $43 \pm 5$ & 275 \\
V2 & $62.92 \pm 3.41$ & $63 \pm 10$ & 283 \\
V1 & $62.03 \pm 3.50$ & $33 \pm 3$ & 289 \\
V5 & $59.94 \pm 4.00$ & $29 \pm 3$ & 297 \\
V6 & $59.28 \pm 3.90$ & $27 \pm 2$ & 299 \\
I & $58.56 \pm 4.07$ & $34 \pm 4$ & 300 \\
II & $58.02 \pm 3.77$ & $45 \pm 5$ & 302 \\
aVR & $54.57 \pm 3.69$ & $34 \pm 3$ & 303 \\
\hline
\end{tabular}

*Th $(\mu \mathrm{V})$, "optimal" threshold value for which sensitivity and specificity are balanced.

Table 2 shows that the 2-lead set consisting of just leads III and V3 achieved high ranking comparable to that attained by the best 3-lead sets. Among 3-lead sets, those using only 1 limb lead (either III or aVL) and 2 precordial leads performed better than lead sets using 2 limb leads and 1 precordial lead.

Table 2. Performance of selected 2- and 3-lead subsets of the ML ECG (Lead Groups 2-4).

\begin{tabular}{lllc}
\hline Lead Set & $\begin{array}{l}\text { AUC } \pm \text { SD } \\
(\%)\end{array}$ & $\begin{array}{l}\text { Th } \pm \text { SD } \\
(\mu \mathrm{V})\end{array}$ & Rank \\
\hline III, V3, V6 & $71.29 \pm 3.47$ & $144 \pm 7$ & 2 \\
aVL, V3, V6 & $71.24 \pm 3.49$ & $129 \pm 14$ & 3 \\
III, V3, V5 & $70.90 \pm 3.34$ & $150 \pm 10$ & 5 \\
aVL, V4, V6 & $70.81 \pm 3.56$ & $109 \pm 5$ & 7 \\
III, V3 & $70.72 \pm 3.30$ & $111 \pm 10$ & 8 \\
III, V3, V4 & $70.56 \pm 3.26$ & $150 \pm 15$ & 9 \\
aVL, V3, V5 & $70.55 \pm 3.30$ & $129 \pm 14$ & 10 \\
III, V4, V6 & $70.54 \pm 3.52$ & $123 \pm 7$ & 11 \\
III, V1, V4 & $70.37 \pm 3.54$ & $128 \pm 8$ & 15 \\
III, V2, V5 & $70.26 \pm 3.08$ & $160 \pm 15$ & 16 \\
I, III, V3 & $70.20 \pm 3.45$ & $151 \pm 13$ & 18 \\
III, V4, V5 & $70.19 \pm 3.25$ & $125 \pm 9$ & 19 \\
aVL, V2, V6 & $70.05 \pm 3.49$ & $142 \pm 11$ & 21 \\
III, V1, V3 & $70.03 \pm 3.45$ & $143 \pm 14$ & 22 \\
\hline
\end{tabular}

Tables 3 and 4 show the differences in the AUC values of the alarm strategies requiring all 12 leads of the ML ECG for the two patient groups. Comparing the rankings of the different strategies in these two tables reveals that several alarm strategies trade positions; however, the differences between the AUC values for the first- and last-ranked lead set are not significant.

Table 3. Performance of all 12 leads of the ML ECG (Lead Group 5) for all 88 patients.

\begin{tabular}{lllr}
\hline Lead Set & $\begin{array}{l}\text { AUC } \pm \text { SD } \\
(\%)\end{array}$ & $\begin{array}{l}\text { Th } \pm \text { SD } \\
(\mu \mathrm{V})\end{array}$ & Rank \\
\hline Any 3 leads & $71.44 \pm 3.24$ & $73 \pm 4$ & 1 \\
Any 2 leads & $70.93 \pm 3.42$ & $88 \pm 6$ & 4 \\
2 contiguous & $70.90 \pm 3.31$ & $86 \pm 6$ & 6 \\
ST12 & $70.43 \pm 3.48$ & $564 \pm 36$ & 13 \\
Any 1 lead & $69.87 \pm 3.35$ & $102 \pm 4$ & 29 \\
\hline
\end{tabular}

Table 4. Performance of all 12 leads of the ML ECG (Lead Group 5) for the 45 "responders" only.

\begin{tabular}{lllr}
\hline Lead Set & $\begin{array}{l}\text { AUC } \pm \text { SD } \\
(\%)\end{array}$ & $\begin{array}{l}\text { Th } \pm \text { SD } \\
(\mu \mathrm{V})\end{array}$ & Rank \\
\hline Any 1 lead & $88.81 \pm 3.03$ & $110 \pm 7$ & 4 \\
2 contiguous & $88.19 \pm 3.30$ & $94 \pm 6$ & 16 \\
Any 2 leads & $88.06 \pm 3.43$ & $95 \pm 5$ & 17 \\
ST12 & $87.32 \pm 3.29$ & $638 \pm 58$ & 31 \\
Any 3 leads & $86.41 \pm 3.50$ & $82 \pm 5$ & 51 \\
\hline
\end{tabular}




\section{Discussion}

Two observations transpire from the results: First, reduced lead sets comprising 3 leads (preferably 1 limb lead, either III or aVL, with 2 precordial leads) and even lead sets with only 2 leads (in particular that using III and V3) have the same ability to detect ischemia as the 12lead ML ECG. Second, when all 12 leads of the ML ECG are available, alarm-detection criteria based on $|\mathrm{ST}|$ exceeding the threshold in any 1 lead, any 2 leads, any 2 contiguous leads, or any 3 leads have the same ischemiadetection ability as the criterion based on the ST12 index calculated as $\Sigma|S T|$ from all 12 leads, provided the appropriate threshold values are used (Tables 3 and 4).

Our rankings of the ST alarm strategies, based on the AUC, for subsets of the 12-lead ML ECG are consistent with the current guidelines [1,5] and with recommendations made previously [2,10]. Drew and Krucoff [2] recommended for ST-segment monitoring of patients with acute coronary syndromes that leads III and V3 be used if only 2 leads are available, and leads III, V3, V5 if 3 are available; our Table 2 supports these recommendations.

It is also evident from Table 2 that two 3-lead sets involving lead V1 (which is highly desirable for arrhythmia monitoring) have nearly the same ischemiadetection ability as the set using leads III, V3, V5. These lead sets can be used for detecting both ischemia and cardiac arrhythmias, thus alleviating the concern [2] that less than 12 leads are not sufficient for detecting both ischemia and arrhythmias in the bedside monitoring.

We are aware of results obtained by Klootwijk et al. [11], who found that 12-lead ST monitoring was in their study superior to 3-lead ST monitoring using leads III, V2, V5 in detecting ischemic episodes. In our Table 2 the latter lead set ranks high, based on an ischemic index calculated as $\Sigma|\mathrm{ST}|$ from all 3 constituent leads; the optimal detection performance is achieved with a threshold of $160 \mu \mathrm{V}$. In light of our results it is conceivable that the alarm detection used by Klootwijk et al., "defined as a change of ST-amplitude in one or more leads of at least $\pm 100 \mu \mathrm{V}$ from the baseline ST level" may be improved by using another strategy, such as summing up $|\mathrm{ST}|$ in leads III, V2, V5, and optimizing threshold of this index.

\section{Conclusions}

Our results suggest that various alarm strategies based on the availability of all 12 leads of the ML ECG are nearly equivalent, whether they use criteria based on $|S T|$ exceeding a given threshold in any 1 lead, any 2 leads, any 2 contiguous leads, any 3 leads, or the ST12 index. When all 12 leads of the ML ECG are not available, certain 2-lead (e.g. III, V3) or 3-lead (e.g. III, V3, V6) subsets of the 12-lead ML ECG can be used as substitutes. The advantage is, these 5- or 6-electrode lead sets can detect ischemia as well as the 10-electrode ML ECG. Moreover, two 3-lead sets with nearly the same ST alarm-detection ability as III, V3, V6 (namely III, V1, V3 and III, V1, V4) are suitable for both ST-segment monitoring of ischemia and arrhythmia monitoring.

\section{Acknowledgements}

Support for PTCA study at Dalhousie University was provided by the Heart \& Stroke Foundation of Nova Scotia and by the Canadian Institutes of Health Research.

\section{References}

[1] Crawford $\mathrm{MH}$, et al. ACC/AHA guidelines for ambulatory electrocardiography. J Am Coll Cardiol 1999;34:912-48.

[2] Drew BJ, Krucoff MW. Multilead ST-segment monitoring in patients with acute coronary syndromes: A consensus statement for healthcare professionals. Am J Crit Care 1999;8:372-88.

[3] Wang JY. Noise stress testing for real-time ST segment measurement algorithms: A new methodology. Computers in Cardiology 2000;27:845-8.

[4] Wang JY, Saenz MC, Horáček BM. Comparison of alarm strategies for continuous 12-lead ST segment monitoring. Computers in Cardiology 2003;30:809-12.

[5] Antman EM, et al. ACC/AHA guidelines for the management of patients with ST-elevation myocardial infarction-executive summary. J Am Coll Cardiol 2004;44:671-719.

[6] Horáček BM, Warren JW, Penney CJ, et al. Optimal electrocardiographic leads for detecting acute myocardial ischemia. J Electrocardiol 2001;34(Suppl):97-111.

[7] Mason RE, Likar I. A new system of multiple-lead exercise electrocardiography. Am Heart J 1966;71:196-205.

[8] Hanley JA, McNeil BJ. The meaning and use of the area under a receiver operating characteristic (ROC) curve. Radiology 1982;143:29-36.

[9] Efron B, Tibshirani RJ. An introduction to the bootstrap. Chapman and Hall, New York, 1993.

[10] Mirvis DM, et al. Instrumentation and practice standards for electrocardiographic monitoring in special care units. Circulation 1989;79:464-71.

[11] Klootwijk P, Meij S, van Es GA, et al. Comparison of usefulness of computer assisted 48-h 3-lead with 12-lead ECG ischaemia monitoring for detection and quantitation of ischaemia in patients with unstable angina. Eur Heart $\mathrm{J}$ 1997;18:931-40.

Address for correspondence

John Wang

Philips Medical Systems

3000 Minuteman Road, MS-0455

Andover, MA 01810-1099

USA

E-mail: john.j.wang@philips.com 